\title{
Expression of a Set of Points' Structure within a Specific Geometrical Model
}

\author{
Jean-Luc Mari and Jean Sequeira \\ LSIS Laboratory - LXAO Department \\ Méditerranée University \\ ESIL, Campus de Luminy, case 925, 13288 Marseille cedex 9, France, \\ jlmari@esil.univ-mrs.fr, \\ : http://esil.univ-mrs.fr/ jlmari/
}

\begin{abstract}
We present a new approach based on a multi-layer model to represent the structure of an object defined by a cloud of points. This technique focuses on the ability to take into account both the global characteristics and the local specificities of a complex object, on topological and morphological levels, as well as on the geometric level. To do that, the proposed model is composed of three layers. We call the boundary mesh the external layer, adding a multiresolution feature. We enhance this representation by including an internal structure: the inner skeleton, which is topologically equivalent to the input object. In addition to that, a third layer links the structural entity and the geometrical crust, to induce an intermediary level of representation. This approach, which overcomes the limitations of skeleton based models and free-form surfaces, is applied to classical and medical data through a specific algorithm.
\end{abstract}

\section{Introduction}

The two main classes of approaches to represent a shape are skeleton based models and free-form surfaces, which are manipulated with control points. The first class allows us to determine the structure of an object, limiting the surface considerations. The second class permits to control the boundary of the shape precisely, but often neglects the general vision we expect. Most of the modeling techniques compensate these lacks by adding features which make the underlying model more complex, but none of them really takes into account both the global and the local specificities of the shape.

In this paper, we focus on designing a modeling method which integrates the local geometric characterization of one class, and the ability to represent the topology and the morphology of the other class.

We use this specific model to reconstruct a set of points. On the opposite of classical methods, our aim is not only to characterize the boundary of the related object. Even if this is sufficient to represent the solid, we want a topological and a morphological descriptor of the object, as well as a coherent link between the various structures. To do that, we introduce three layers whose roles are to take into account these features.

In Sect. 2, we skim over different models' formalizations, to extract the key characteristics of our approach. In Sect. 3. we describe the principle of a new modeling approach. We define the model by developing its three main entities: the inner skeleton, 
the external layer and the transition layer. Then we detail the reconstruction process itself in Sect.4 We finally validate the approach with examples (one of them being from real medical data) in Sect. 5]

\section{Overview}

Implicit Surfaces and Skeletons. Most of the skeleton based techniques use the formalism of implicit surfaces. These surfaces, whose skeleton is usually a set of geometrical primitives, have been more and more employed in computer graphics over the past 15 years. These approaches have several advantages, such as providing a compact and volume-based representation of the object. Moreover, the skeleton supplies the topological prior assumption and the structural information. However these surfaces are rather dedicated to represent smooth objects, sharp edges being more difficult to obtain. Moreover, the characterization of details implies to take into account a large number of primitives. These arguments point out the fact that it is difficult to get a local control with such surfaces because of the growing number of primitives to consider.

Surfaces Defined by Control Points. The most common formalism to represent a freeform object consists in using a parametric surface. Among these kinds of surfaces, defined by control points, there are the classical Bézier, B-splines and NURBS. The control points imply an intuitive and precise appreciation of the shape to model. Particularly, it is for this reason these surfaces are frequently used in CAD/CAM: they permit an intrinsic local control on the object's geometry. However, the modeling of objects with complex topologies (like with branching shapes or holes) keeps on being a traditional problem. Moreover, this kind of representation is hard to apprehend on a global level. If we wish to deform an initial model by stretching it, we have to move the right set of points, and to verify the induced transformations on the shape. Although high level operators exist to solve this problem (like warping), these manipulations are fussy and hard to calibrate (taking into account a small set of points or the whole object).

Similar Techniques. Among the approaches that emerge from the problematics to take into account both global and local characteristics of a 3D object, there are hierarchical $B$-splines (H-splines) [1], simplex meshes [2] and enhanced implicit methods (skins [3]). Multiresolution techniques are frequently used according to the complexity of the geometrical details and the size of the data. Most of the time, it consists in representing the object within several levels of detail [4,56]. In this frame, the formalism of subdivision surfaces is more and more used, as it presents a powerful multiresolution feature. It is nowadays used into a large panel of applications in computer graphics [7]8].

An Interesting Compromise. The approaches with skeletons show that instead of considering the skeleton's instability, we should take into account that it is not well adapted to surface phenomena, but rather to shape description. The control points approaches provide a very good control on differential properties, but hardly any on topology and morphology. We need a model that integrates in a coherent way the global and local characteristics of these two approaches. 


\section{The Multilayer Model}

The model must allow us to control these three concepts in a coherent framework: the topology (to be able to model complex shapes with no prior assumption), the morphology (including a shape descriptor) and the geometry (integrating a crust entity for precise handling). Moreover, according to the size of the data to model, we wish to include the multiresolution feature. We can define the aims of our modeling approach in two points: we want both the global structure and the boundary surface; and we want to detach the surface representation from the global shape's.

The model is composed of three layers (see Fig. 1 1 a). The first layer, the internal one, that we call inner skeleton (layer $L_{I}$ ). It defines the global structure of the shape, on topological and morphological levels. The external layer (layer $L_{E}$ ) characterizes the local variations of the shape's surface, regardless of the skeleton. The transition level $L_{T}$ represents the articulation between the internal and external layers.

The goal of this split between local and global characterization is that the local perturbations on the surface do not deteriorate the global shape descriptor which is the inner skeleton, and that the transformations on this inner structure are propagated on the external layer.

Inner Skeleton. The inner skeleton $L_{I}$ is a homotopic kernel enhanced by morphological features. We define the inner skeleton $L_{I}$ on a structural level as a 3-complex, i.e. a set of tetrahedrons, triangles, segments and points. The edges define the connectedness relations between vertices. When three neighbors are connected, we obtain a triangle, and when four neighbors are connected, we obtain a tetrahedron (Fig.11-b). A cycle of edges defines a one-holed surface.
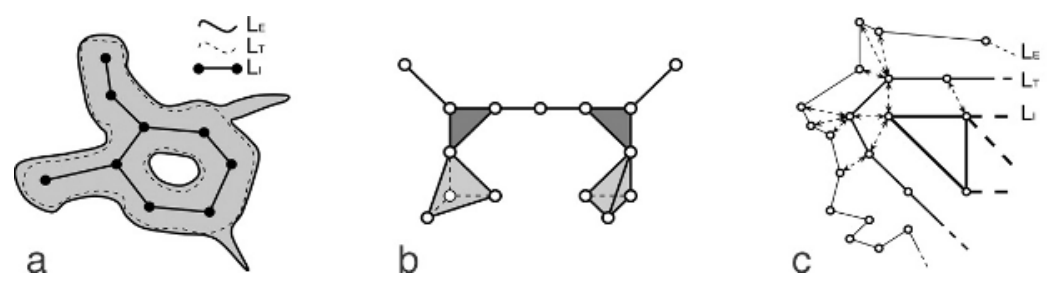

Fig. 1. (a) Scheme of the 3-layer model. (b) Example of 3D structure for the inner skeleton. (c) The three layers and the links between the primitives

External Layer. The external layer $L_{E}$ is a simple triangulation. The vocation of this layer is to define the geometry of the object, given as a set of unstructured points. The multiresolution feature is supported by this layer. Considering $L_{E}^{100 \%}$ the maximal level of detail for this layer, the various resolution levels are defined by $L_{E}^{r \%}$, with $r_{\min } \leq r \leq 100$, $r_{\text {min }}$ being the percentage below which the layer becomes non-manifold.

Transition Layer. The transition layer $L_{T}$ represents an intermediate geometrical level and a structural entity which makes the link between the global definition and the local characterization of an object. The inner and external representation levels are both as important and we want to characterize the articulation between them. 
In the geometrical frame, we define the transition layer as an intermediary triangulation between the two other entities. It induces a structural link allowing us to go from one layer to another (i.e. an element of the external layer can refer to an element of the inner layer and vice versa). Furthermore, we set the equality $L_{T}=L_{E}^{r_{\min } \%}$ between the transition layer and the minimal resolution level of the external layer. The fact that $L_{T}$ is the most simplified level of $L_{E}$ provides a natural evolution from $L_{E}^{100 \%}$ to the transition layer $L_{T}$ by mesh reduction in the reconstruction process.

Transition Graph. In addition to the previous geometrical definition, the transition layer includes a particular data structure: the underlying graph $G_{T}$ linking the two skeletons allows us to set coherent relations within the object (see Fig. 1. c). The edges of this graph are defined by a shortest distance criterion [9].

\section{Reconstruction}

In this section, we develop the reconstruction process related to the proposed model in order to express how the three layers are obtained, starting from a 3D set of points.

The main idea is to get an expression of the structure of an unorganized cloud of points, given as input data. We do not simply want to characterize the boundary of the shape with the external layer. Even if it is sufficient to represent the related solid, we attempt to exhibit a topological and morphological descriptor of the object. This point is fundamental, because efficient techniques of reconstruction are numerous, but they principally focus on the surface reconstruction without taking the structure into account.

\subsection{Specific Process}

The process is composed of two stages, themselves being most of the time well known techniques in computer graphics. However, concerning the first stage, we developed an original method to obtain the inner skeleton by doing an homotopic peeling applied to an octree.

The following algorithm consists of two independent steps: the extraction of the inner skeleton and the characterization of the crust (from the external layer to the transition layer). We illustrate the process by considering the Stanford bunn) 1 data in the initial form of a cloud of points (cf. Fig. 2-a) . It is a simple object, of genus zero, but more complex examples are given in the next section.

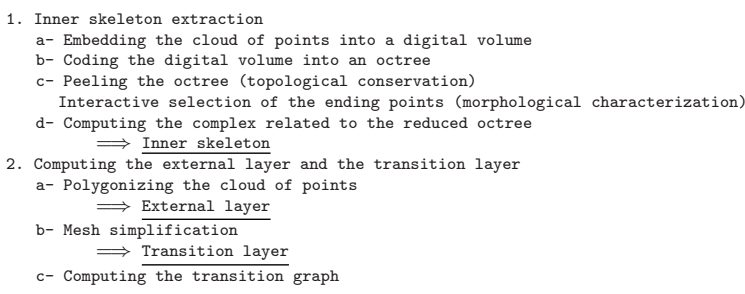

${ }_{1}^{1}$ available at the URL: http//www-graphics . stanford.edu/data/ 


\subsection{Inner Skeleton Extraction}

The inner skeleton extraction goes through a conversion of the data into the discrete space $\mathbb{Z}^{3}$. We wish to characterize the topology and the global shape, by keeping only a small set of relevant voxels (or groups of voxels).

Step 1: Embedding the Cloud of Points into a Digital Volume. Let $C$ be the initial cloud of points. We adopt the same principle found in [10] to embed $C$ into a digital volume $V$ (Fig. 2,b).

Step 2: Octree Conversion. The digital volume is then converted into an octree [11], to keep in mind the notion of voxels grouping. A single voxel does not represent an important morphological detail, but on the contrary, a block of voxels defines a large area that has to be included into the structure of the inner skeleton (Fig.2-1).

Step 3: Interactive Thinning of the Octree. We adopt a classical thinning process, whose asset is to supply a homeomorphic entity related to the object. There is no algorithm dealing with octrees in such a way, so we use a simple algorithm initially designed for digital volumes, and we adapt it to work on octrees (by modifying the neighborhood relationship and the local thinning criterion). The thinning (or the peeling) problematics first appeared in $\mathbb{Z}^{2}[12]$. The principle is to delete the pixels that do not affect to topology of the object. Such points are said simple: when erased, no holes are created and no components are disconnected. [13] extended this concept to $\mathbb{Z}^{3}$. To extend this criterion to an octree, we defined in [9] the $\omega$-neighborhood as the equivalent to the 26neighborhood in $\mathbb{Z}^{3}$, and the $\bar{\omega}$-neighborhood as the equivalent to the 6-neighborhood (for the complementary object, i.e. the background).

We expose the algorithm used to peel an octree, which is derived from the initial thinning process into a digital volume. In our case, the size of the octree elements intervenes.

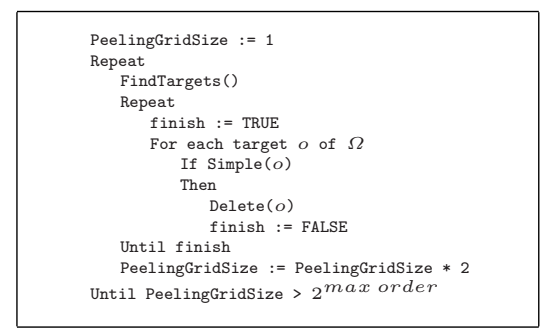

The routine FindTargets () computes the list of the octree elements of the object whose size equals the current peeling grid size (i.e. elements that can be erased). The Figs.2-d,e illustrate two steps of the bunny peeling with this algorithm. We enhance this algorithm by adding the interactive feature that allows the user to guide the process. It consists in setting representative elements, which contribute to the morphology as ending points. When the current element is such a point, it cannot be removed. The Figs.2-f,g show the result when four ending points are selected by the user: the ears, the nose and the tail. The resulting octree is really homeomorphic to the initial object, and it supplies a good morphological characterization. 
Step 4: Computing the Complex Related to the Reduced Octree. The last step to get the inner skeleton $L_{I}$ consists in computing the reduced octree into a complex. This is done thanks to the $\omega$-neighborhood. Edges, triangles and tetrahedrons are created according to the adjacency of the octree elements (Fig.2-h).
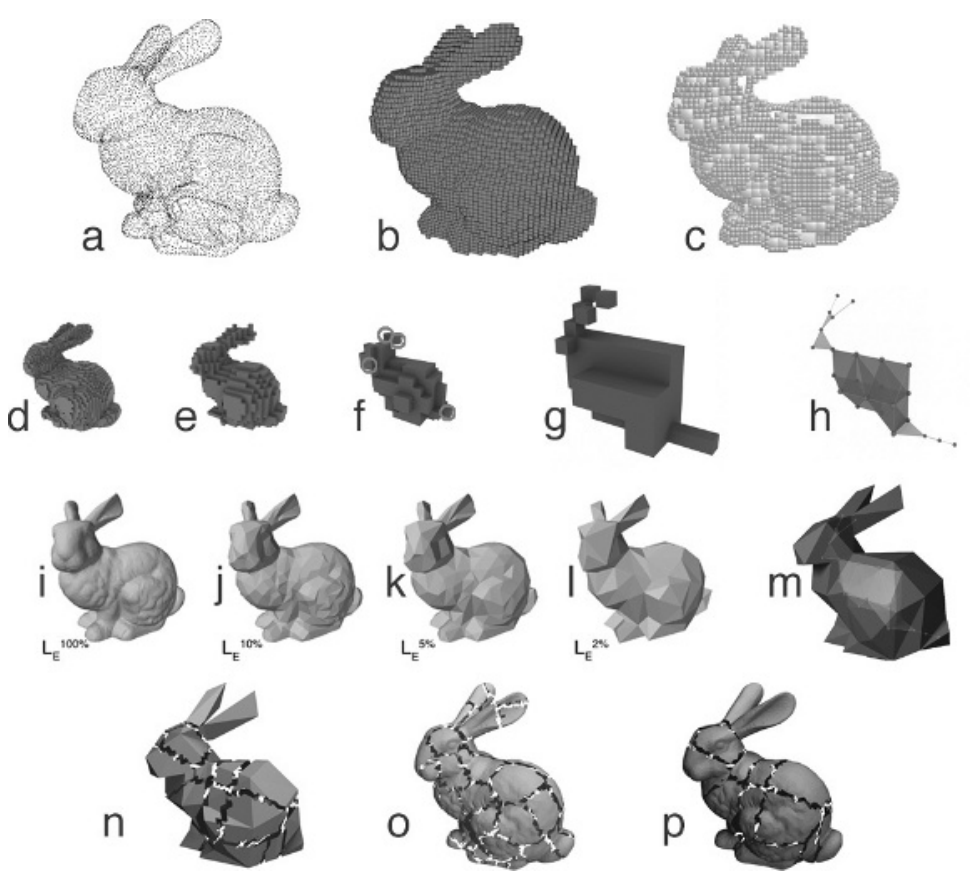

Fig. 2. (a) Initial cloud of points. (b) Digital volume. (c) Octree of the bunny. (d,e) Two peeling steps. (f,g) Setting four ending points. (h) Inner skeleton. (i,j,k,l) Some LoDs of the bunny. (m) The transition layer of the bunny. (n,o,p) Distribution of the points between the three layers

\subsection{From the External Layer to the Transition Layer}

Step 1: Polygonizing the Cloud of Points. The finest geometrical characterization of the external layer $L_{E}^{100 \%}$ is a classical triangulation of the data points (see Fig.22-i). We used the Cocone module described in [14].

Step 2: Multiresolution and Transition Layer. The Figs. 2- $\mathrm{j}, \mathrm{k}, 1$ show some reduced meshes of the bunny, until $L_{E}^{1 \%}$ which is the last step before the triangulation becomes non-manifold (cf. Fig. $2 \mathrm{rm}$ ). We go from the external to the transition layer, and we set $L_{T}=L_{E}^{1 \%}$ to define the geometrical characterization of the transition layer. All the mesh simplifications in this paper have been done using QSlim module [5].

Step 3: Computing the Transition Graph. To make the link between structural and geometrical levels of the model, the last step of the whole process is the computation of the transition graph $G_{T}$. The Figs. 2fn,o,p show the distribution of the points of $L_{T}$ 
according to the points of $L_{I}(\mathrm{n})$, the points of $L_{E}$ according to the points of $L_{T}$ (o), and the points of $L_{E}$ according to the points of $L_{I}$ (p).

\section{Validation and Examples}

In addition to the bunny's example, we go further into the validation of our approach by taking three other examples. The horse and the dragon (a one holed object) are classical clouds of points from the Stanford database, and the last example comes from medical imaging (a foetus's heart). Such an organ presents a complex structure. The table below illustrates the number of vertices according to the layers for each example. The Fig. 3 shows the inner skeleton, the transition layer and the external layer of the three objects.

\begin{tabular}{|c|c|c|c|}
\hline Number of vertices & $L_{I}$ & $L_{T}$ & $L_{E}^{100 \%}$ \\
\hline Bunny & 24 & 76 & 7030 \\
\hline Horse & 97 & 244 & 48485 \\
\hline Dragon & 63 & 108 & 44315 \\
\hline Heart & 1310 & 1596 & 35260 \\
\hline
\end{tabular}
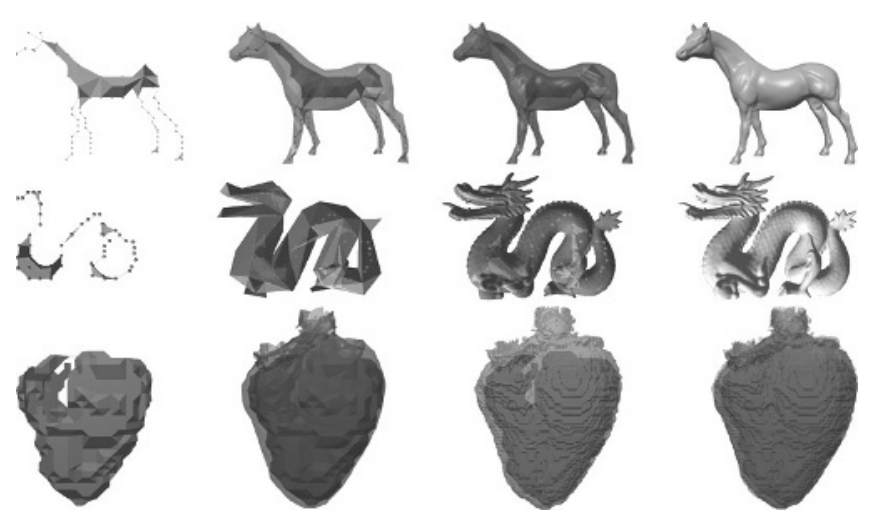

Fig. 3. Expression of the structure of four objects within the reconstruction process

\section{Future Work}

At the current level of the approach's elaboration, we envisage several points which we feel are necessary to be dealt with as future work.

The good morphological properties of the inner skeleton could be used in a shapes' recognition module. The aim being to classify an object on topological and morphological criterions, the process could lie on a catalog of typical objects arranged according to shape indications. This analysis perspective could be applied to computer vision, robotics, etc.

The major work to do in the future is the animation of a reconstructed object. This can only be done if the object is well positioned (as an evidence, it cannot work on the bunny, as legs are not defined by the inner skeleton). For example, to animate a character 
expressed by the 3-layer model in a standing position (limbs being well defined), specific rules have to be determined to move external and transition layers after handling the skeleton.

\section{Conclusion}

We have presented a new shape formalism, which aims to give an explicit expression of an unstructured cloud of points, through three entities. The external layer defines the crust of the object in a classical way. In addition to that, the transition layer and, above all, the inner skeleton, allow us to get the structure of the object. This is done not only by characterizing the global shape, but also by a specifying a relevant topological entity. Moreover, we have validated our approach on typical data and on complex medical data. We believe the underlying model can be applied to several applicative domains, taking part of the inner skeleton's assets.

\section{References}

1. D. R. Forsey, R. H. Bartels: Hierarchical B-spline refinement. Computer Graphics (Proceedings of SIGGRAPH'88) (1988) 22:4 205-212

2. H. Delingette: Simplex Meshes: a general representation for 3D shape reconstruction. INRIA Reseach Report 2214 (1994)

3. L. Markosian, J. M. Cohen, T. Crulli, J. Hugues: Skin: a constructive approach to modeling free-form shapes. Computer Graphics Proceedings (SIGGRAPH'99) (1999) 393-400

4. W.J. Schroeder, J.A. Zarge, W.E. Lorensen: Decimation of triangle meshes. ACM Computer Graphics (SIGGRAPH '92 Proceedings) 26 (1992) 65-70

5. M. Garland, P.S. Heckbert: Surface Simplification Using Quadric Error Metrics: Computer Graphics (Annual Conference Series) 31 (1997) 209-216

6. M. Eck, T. DeRose, T. Duchamp, H. Hoppe, M. Lounsbery, W. Stuetzle: Multiresolution analysis of arbitrary meshes. SIGGRAPH'95 Proceedings (1995) 173-181

7. M. Lounsbery, T. DeRose, J. Warren: Multiresolution analysis for surfaces of arbitrary topological type. ACM Transactions on Graphics 16:1 (1997) 34-73

8. A. Lee, W. Sweldens, P. Schrфder, L. Cowsar, D. Dobkin: MAPS: Multiresolution Adaptive Parameterization of Surfaces. Computer Graphics Proceedings (SIGGRAPH'98) (1998) 95104

9. J.-L. Mari: Modélisation de formes complexes intégrant leurs caractéristiques globales et leurs spécificités locales. PhD Thesis, Université de la Méditérranée - Aix-Marseille II (France) (2002). http://www.esil.univ-mrs.fr/ jlmari/

10. E. Bittar, N. Tsingos, M.-P. Gascuel: Automatic reconstruction of unstructured 3D data: Combining a medial axis and implicit surfaces. Computer Graphics Forum (Eurographics'95 Proc.) 14 (1995) 457-468

11. D. Meagher: Geometric modeling using octree encoding. IEEE Computer graphics and Image Processing 19:2 (1982) 129-147

12. A. Rosenfeld: A characterization of parallel thinning algorithms. Information Control 29 (1975) 286-291

13. G. Bertrand: Simple points, topological numbers and geodesic neighborhoods in cubic grids. Patterns Recognition Letters 15 (1994) 1003-1011

14. N. Amenta, S. Choi, T.K. Dey, N. Leekha A simple algorithm for homeomorphic surface reconstruction. 16th ACM Symposium on Computational Geometry (2000) 213-222 